\title{
Modification of the PTCDA-Ag bond by forming a heteromolecular bilayer film
}

\author{
Benjamin Stadtmüller, ${ }^{1,2,{ }^{*}}$ Martin Willenbockel, ${ }^{1,2}$ Sonja Schröder, ${ }^{1,2}$ Christoph Kleimann, ${ }^{1,2}$ Eva M. Reinisch, ${ }^{3}$ \\ Thomas Ules, ${ }^{3}$ Sergey Soubatch, ${ }^{1,2}$ Michael G. Ramsey, ${ }^{3}$ F. Stefan Tautz, ${ }^{1,2}$ and Christian Kumpf ${ }^{1,2}$ \\ ${ }^{1}$ Peter Grünberg Institut (PGI-3), Forschungszentrum Jülich, 52425 Jülich, Germany \\ ${ }^{2}$ Jülich-Aachen Research Alliance (JARA) - Fundamentals of Future Information Technology, 52425 Jülich, Germany \\ ${ }^{3}$ Institut für Physik, Karl-Franzens-Universität Graz, 8010 Graz, Austria \\ (Received 15 December 2014; revised manuscript received 5 March 2015; published 28 April 2015)
}

\begin{abstract}
The understanding of the fundamental physical properties of metal-organic and organic-organic interfaces is crucial for improving the performance of organic electronic devices. This is particularly true for (multilayer) systems containing several molecular species due to their relevance for donor-acceptor systems. A prototypical heteromolecular bilayer system is copper-II-phthalocyanine $(\mathrm{CuPc})$ on 3,4,9,10-perylene-tetra-carboxylicdianhydride (PTCDA) on $\operatorname{Ag}(111)$. In an earlier work we have reported a commensurate registry between both organic layers and an enhanced charge transfer from the Ag substrate into the organic bilayer film [Phys. Rev. Lett. 108, 106103 (2012)], which both indicate an unexpectedly strong intermolecular interaction across the organic-organic interface. Here we present new details regarding electronic and geometric structure for the same system. In particular, we provide evidence that the enhanced charge transfer from the substrate into the organic bilayer does not involve CuPc electronic states, hence, there is no significant charge transfer into the second organic layer. Furthermore, we report vertical bonding distances revealing a shortening of the PTCDA-Ag(111) distance upon $\mathrm{CuPc}$ adsorption. Thus, electronic and geometric properties (charge transfer and bonding distance, respectively) both indicate a strengthening of the PTCDA-Ag(111) bond upon CuPc adsorption. We explain these findings - in particular the correlation between $\mathrm{CuPc}$ adsorption and increased charge transfer into PTCDA-in a model involving an intermolecular screening mechanism.
\end{abstract}

DOI: 10.1103/PhysRevB.91.155433

PACS number(s): 68.43.Fg, 79.60.Dp, 68.49.Uv

\section{INTRODUCTION}

The interest in organic adsorbates on metal surfaces is not only inspired by the large potential of this class of materials for electronic devices, such as light emitting diodes or organic photovoltaic cells, but also by the great relevance of the fundamental interaction mechanisms occurring at the interface between different materials. A comprehensive understanding of the physical properties of metal-organic interfaces was achieved by studying the adsorption of the organic interface layer of prototype molecules, such as 3,4,9,10-perylenetetra-carboxylic-dianhydride (PTCDA) [1-6], metal phthalocyanines (MePc) [7-16], and other $\pi$-conjugated molecules [17-20] on highly crystalline (noble) metal surfaces. In many investigations it was found that the geometric structure and the electronic level alignment at these metal-organic interfaces are determined by the interactions between the molecules themselves, as well as between the molecules and the metal surface. In particular, the charge transfer from the metal substrate into the lowest unoccupied molecular orbital (LUMO), which is the signature of the chemical interaction between the molecule and the surface, is always reflected by the adsorption height of the molecule on the surface [5,6,21-23]. The latter can therefore be seen as a geometric fingerprint of the bonding strength.

While such homomolecular adsorbate systems are rather well understood, comparably little is known about the interaction mechanisms at heteromolecular interfaces, i.e.,

\footnotetext{
*bstadtmueller@physik.uni-kl.de; Present address: Department of Physics and Research Center OPTIMAS, University of Kaiserslautern, Erwin-Schroedinger-Strasse 46, 67663 Kaiserslautern, Germany.
}

about interfaces between layers containing different types of molecules. It is commonly believed-and supported by many experimental results reported so far [24-28] - that the interaction between different organic materials is dominated by weak van der Waals forces, as it is the case in organic bulk crystals. However, there is some recent evidence for two adsorbate systems showing a stronger interaction across a heteromolecular interface $[29,30]$. In both cases this is indicated by a commensurate registry between the two molecular layers, which forces the molecules of the second layer in an artificial and unnatural structure. One system consists of layers of fluorinated and nonfluorinated copperII-phthalocyanine molecules $\left(\mathrm{F}_{16} \mathrm{CuPc} / \mathrm{CuPc} / \mathrm{Ag}(111)\right)$ [29], the other is $\mathrm{CuPc} / \mathrm{PTCDA} / \mathrm{Ag}(111)$. For the latter, which is also the topic of the present work, ultraviolet photoelectron spectroscopy (UPS) experiments indicated that the intermolecular interaction is possibly not purely van der Waals-like [30].

When $\mathrm{CuPc}$ molecules are deposited on a closed monolayer of PTCDA/ $\operatorname{Ag}(111)$ at room temperature, they form a homogeneous two-dimensional (2D) gas floating on the PTCDA monolayer with a maximized lateral distance between the molecules that decreases continuously with increasing $\mathrm{CuPc}$ coverage [30]. This behavior is very similar to the adsorption directly on the $\operatorname{Ag}(111)$ surface [11]. Completing the first CuPc layer on PTCDA, or decreasing the sample temperature to $160 \mathrm{~K}$ or below, results in a phase transition from the disordered $\mathrm{CuPc}$ film to an ordered, commensurate superstructure, again revealing a very similar behavior to the direct adsorption on $\operatorname{Ag}(111)$. The commensurate registry between $\mathrm{CuPc}$ and PTCDA lattices indicates a site-specific interaction between the organic layers and proves the dominant influence of the PTCDA layer on the structure formation of the $\mathrm{CuPc}$ film [30]. In addition, the electronic valence structure of the PTCDA monolayer film is significantly altered by the 
adsorption of CuPc. For the mere PTCDA layer the LUMO is already partially occupied due to the interaction with the $\mathrm{Ag}(111)$ substrate. In an UPS experiment a corresponding former-LUMO (FLUMO) peak appears close to the Fermi energy $E_{F}$ and has a very asymmetric shape since it is cut by the Fermi edge. This peak, called Fermi-level peak in the following, shifts continuously to larger binding energies, when an increasing amount of CuPc is deposited on the PTCDA layer (for details see Ref. [30], in particular Fig. 3). However, it is a priori unclear whether this shift indicates just an increasing population of the PTCDA LUMO, or additionally a filling of the CuPc LUMO. Neither the UPS nor any structural data (including the interlayer spacing between the $\mathrm{CuPc}$ and PTCDA layers, which was determined to be $3.22 \AA$ by normal incidence X-ray standing waves, see below and Ref. [30]) can answer this question unambiguously.

The fundamental question addressed in this paper is the nature of the Fermi-level peak shift induced by the $\mathrm{CuPc}$ adsorption on PTCDA/Ag(111). This issue could not be answered in our earlier work. The present paper, besides providing more detailed information regarding the data analysis, addresses this aspect by investigating how both electronic and geometric structure are affected by the additional organic layer. First, we performed an angle-resolved photoelectron spectroscopy (ARPES) study, which was analyzed using the orbital tomography approach [31,32]. This allows us to identify the emitting molecular orbitals at each binding energy by means of the angular distribution pattern of their photoemission yield [33], and results in the projected density of states (PDOS) for each orbital of any inequivalent molecule on the surface. In our case the LUMO orbitals of both molecular species are of particular interest. We find that the CuPc LUMO is not involved in the modification of the photoelectron yield close to $E_{F}$, hence the Fermi-level peak shift is only caused by an additional filling of the PTCDA LUMO. This indicates that there is no charge transfer between the two organic layers. Second, results and experimental details of normal incidence $\mathrm{X}$-ray standing wave (NIXSW) studies are discussed. We determine the adsorption height of atomic species within the molecular adsorbates with an accuracy better than $\approx 0.05 \AA$. We find that the PTCDA layer approaches the Ag surface when $\mathrm{CuPc}$ is adsorbed on top. This shortening of the bonding distance agrees well with the increasing charge transfer into the PTCDA LUMO. We develop a model involving intermolecular screening, which explains the relation of the adsorption height change, the PTCDA LUMO shift and the CuPc adsorption.

\section{EXPERIMENTAL DETAILS}

\section{A. Sample preparation}

All experiments as well as the sample preparations were performed under identical conditions as for the experiments reported in Ref. [30]. The base pressure in the ultra-high-vacuum systems was always below $8 \times 10^{-10}$ mbar. The surface of the (111)-oriented silver crystal was cleaned by repeated cycles of argon ion bombardment and subsequent annealing at a temperature of $T_{\text {sample }}=730 \mathrm{~K}$. The cleanliness of the $\operatorname{Ag}(111)$ surface was verified either by searching for contaminations in core-level spectroscopy or by measuring the surface state at the $\bar{\Gamma}$ point of the surface Brillouin zone using ARPES.
For deposition of the organic material we used a dedicated evaporator system and kept the sample at room temperature. The heteromolecular bilayer films were always prepared in two subsequent steps: At first thick PTCDA films (3-5 monolayers, ML) were deposited onto the clean $\operatorname{Ag}(111)$ crystal, followed by sample annealing at the desorption temperature of the PTCDA bilayer $(\approx 590 \mathrm{~K})$. This procedure is known to result in a closed PTCDA monolayer film, well ordered with the known herringbone structure [34]. Afterwards, CuPc was deposited with a constant deposition rate of $\approx 0.15 \frac{\mathrm{ML}}{\mathrm{min}}$ that was monitored with a quadrupole mass spectrometer (QMS). For determining the CuPc coverage we integrated the QMS signal over the deposition time. This procedure for the $\mathrm{CuPc}$ coverage determination has been calibrated beforehand in a series of $\mathrm{CuPc} / \mathrm{Ag}(111)$ preparations, whereby the monolayer coverage was defined by the highest coverage obtained, which did not yet show any bilayer signal in thermal desorption spectroscopy. During all experiments, we carefully checked for radiation damage and adjusted the acquisition time of all ARPES and XSW scans accordingly.

\section{B. Angle-resolved photoelectron spectroscopy and orbital tomography}

ARPES experiments were performed at beam line U125/2SGM of the BESSY II storage ring at the Helmholtz Zentrum Berlin. The sample was illuminated by monochromatic radiation with the energy $\hbar \omega=35 \mathrm{eV}$ at a fixed angle of incident of $40^{\circ}$ to the surface normal. The photoelectron yield was recorded with a toroidal electron analyzer having a polar acceptance angle of $\pm 80^{\circ}$ and an energy dispersion range of $1.0 \mathrm{eV}$ at a pass energy of $10 \mathrm{eV}$. For these experimental parameters the energy resolution was estimated from the broadening of the Fermi edge at RT to be better than $150 \mathrm{meV}$. The azimuthal angular distribution was recorded by rotating the sample around its surface normal in steps of $1^{\circ}$ in a range of at least $130^{\circ}$. The measured data was converted from polar to cartesian coordinates considering the threefold symmetry of the data, and resulting in a three-dimensional ARPES data cube $I\left(k_{x}, k_{y}, E_{B}\right)$. Constant binding energy (CBE) maps, as they will be discussed in the following, represent two-dimensional cuts through this data cube at a fixed binding energy $E_{B}$.

Within the orbital tomography approach we employ the function

$$
\begin{aligned}
F\left(k_{x}, k_{y}, E_{B}\right)= & \sum_{i} a_{i}\left(E_{B}\right) \Phi_{i}\left(k_{x}, k_{y}\right) \\
& +b\left(E_{B}\right) I_{s u b}\left(k_{x}, k_{y}, E_{B}\right)+c\left(E_{B}\right)
\end{aligned}
$$

in order to model the ARPES data cube. It represents a linear combination of theoretical CBE maps $\Phi_{i}\left(k_{x}, k_{y}\right)$, which are calculated for all molecular orbitals $i$ in question, and includes a substrate contribution $I_{s u b}\left(k_{x}, k_{y}, E_{B}\right)$ [with its amplitude $\left.b\left(E_{B}\right)\right]$ as well as a constant offset function $c\left(E_{B}\right)$. The theoretical CBE maps $\Phi_{i}\left(k_{x}, k_{y}\right)$ are calculated under the assumption of a plane-wave final state (for more details see Refs. [31-33]). The fitting parameters $a_{i}\left(E_{B}\right)$ represent the main result of the orbital tomography approach. They are functions of the binding energy $E_{B}$, and can therefore be understood as the energy-resolved density of states projected on the 
corresponding molecular orbital (PDOS). Further details can be found in Refs. [31] and [32].

\section{Normal incidence $x$-ray standing waves}

The NIXSW experiments were carried out at the UHV end station of beam line ID32 at the European Synchrotron Radiation Facility (ESRF) in Grenoble, France. This end station was equipped with a hemispherical electron analyzer (PHOIBOS 225, SPECS) mounted perpendicular to the incoming photon beam, and with all the equipment that is necessary for sample preparation and precharacterization using LEED.

NIXSW allows us to determine the vertical distance of atomic species within an adsorbate system to the surface of a substrate single crystal with very high precision (typically $<0.05 \AA$ ). Since the method is chemically sensitive, all chemically different atomic species can be probed independently, which enables, e.g., the detection of molecular bending or the separate analysis of functional groups. Here, we only very briefly summarize the fundamental aspects of this method, more details can be found, e.g., in Refs. [35] or [36].

For a photon energy and geometry, which fulfill the Bragg condition $\vec{H}=(h k l)=\vec{k}_{H}-\vec{k}_{0}$ for a certain substrate Bragg reflection, an $\mathrm{x}$-ray standing wave field is formed by the interference of the incoming $\vec{E}_{0}$ and the Bragg-reflected wave $\vec{E}_{H}$. Scanning the photon energy through the Bragg condition results in a shift of the phase difference $v$ of incoming and Bragg reflected waves by $\pi$ and therefore shifts the standing wave field through the crystal by half a lattice spacing $d_{h k l}$ in the direction perpendicular to the Bragg planes. Consequently the photon density at a certain position $D^{H}$ above the surface (more precisely: above the lattice planes of the $\vec{H}$ reflection) changes as a function of the photon energy, which in turn influences the absorption of an atom located at that height. The absorption can be monitored by recording the partial core-level yield $I(E)$ of the photoelectrons stemming from the specific atomic species. This experimental yield curve $I(E)$ can be modeled by $[35,36]$

$$
I(E)=1+R(E)+2 \sqrt{R(E)} F^{H} \cos \left(v(E)-2 \pi P^{H}\right) .
$$

$R(E)$ is the reflected intensity with its complex amplitude $\sqrt{R(E)}$ and phase $v(E)$. The actual fitting parameters are the coherent position $P^{H}$ and the coherent fraction $F^{H}$. The coherent position $P^{H}$ represents the averaged vertical position $D^{H}$ of all chemically equivalent atoms in question, modulo the lattice-plane spacing $d_{H}$ of the substrate crystal, and in units of $P^{H}=\left(D^{H} \bmod d_{H}\right) / d_{H}$. The coherent fraction $F^{H}$ can be understood as a vertical ordering parameter obtaining values between 0 and 1 , whereby $F^{H}=0$ indicates complete disorder (in the sense of a homogeneous distribution of vertical adsorption heights) while for $F^{H}=1$ all emitting atoms are located at the same position $P^{H}$.

\section{EXPERIMENTAL RESULTS}

\section{A. Electronic structure}

The results of our UPS study of the electronic valence structure of the heteromolecular bilayer system $\mathrm{CuPc/PTCDA} / \operatorname{Ag}(111)$ have been discussed in Ref. [30]. The
UPS data (see Fig. 3 of Ref. [30]) are dominated by three spectroscopic features, the PTCDA and CuPc HOMO peaks, and a "Fermi-level peak" located directly below the Fermi level. The assignment of the first two peaks to the HOMOs of the molecules is unambiguous. Note that the binding energy of the PTCDA HOMO is hardly affected by the adsorption of $\mathrm{CuPc}$, and that the $\mathrm{CuPc} \mathrm{HOMO}$ reveals its well known asymmetric line shape [37]. The third peak is attributed to the PTCDA LUMO, which becomes partially filled due to the interaction with the substrate already before the CuPc is adsorbed. But most remarkably, this peak shifts continuously towards larger binding energies when the $\mathrm{CuPc}$ molecules are deposited. A maximum shift of $120 \mathrm{meV}$ corresponds to 1.0 ML of CuPc. This rather large shift indicates a modification of the charge distribution in the heteromolecular bilayer film, and possibly even a hybridization of $\mathrm{CuPc}$ and PTCDA (LUMO) states, as speculated in Ref. [30].

In order to determine the nature of this third UPS peak (and verify the origin of the others), we have recorded ARPES data in the valence region for a heteromolecular bilayer film with a $\mathrm{CuPc}$ coverage of $0.7 \mathrm{ML}$. The resulting experimental $\mathrm{CBE}$ maps are shown in Figs. 1(a), 1(c), and 1(e) for the relevant binding energies, which are known from the UPS data [30]. Corresponding CBE maps, based on the assignment of the spectroscopic features discussed in the previous paragraph, are shown in the lower row [Figs. 1(b), 1(d), and 1(f)]. Note that the theoretical CBE maps for the PTCDA orbitals [Fig. 1(b) and 1(f)] have been discussed previously in Ref. [32]. They take into account the emission from two inequivalent PTCDA molecules, which occur in the unit cell of the PTCDA/Ag(111) herringbone structure, as well as all rotational and mirror domains due to the $p 3 m 1$ symmetry of the substrate surface. As a result, both orbital emission patterns reveal a sixfold symmetry and pronounced maxima at a well-defined distance from the $\bar{\Gamma}$ point of the surface Brillouin zone. In contrast, the calculated CBE map for the CuPc HOMO (and LUMO, see below) reveals just a homogeneous ring of intensity in momentum space [see Fig. 1(d)]. This is due to the rotational disorder of the CuPc molecules in the diluted 2D gas, which is formed at this $\mathrm{CuPc}$ coverage.

Note that the experimental CBE maps at $E_{B}=1.58 \mathrm{eV}$ and $E_{B}=0.90 \mathrm{eV}$ [Figs. 1(a) and 1(c)] match the calculated emission patterns of the HOMOs of both molecules (b) and (d) very well. This confirms the assignment of UPS peaks and orbitals discussed above and in Ref. [30], and proves that the in-plane orientation of the PTCDA molecules is not altered by the adsorption of $\mathrm{CuPc}$ in the second layer. However, the experimental $\mathrm{CBE}$ map of the frontier orbital of the CuPc/PTCDA bilayer film at $E_{B}=0.27 \mathrm{eV}$ [Fig. 1(e)] does not completely fit the corresponding prediction for a pristine PTCDA monolayer structure (f). Although both CBE maps show six clear maxima at the same momentum space positions, in the experimental data three maxima are more pronounced and lead to a threefold symmetry. The quantitative analysis presented in the following shows that this is due to the substrate contribution to the ARPES emission, which is more prominent in this energy range rather than in that of the HOMOs.

We have analyzed the ARPES data quantitatively using the orbital tomography approach. The resulting PDOS of the relevant molecular orbitals are displayed in Figs. 2(a) and 2(b), 


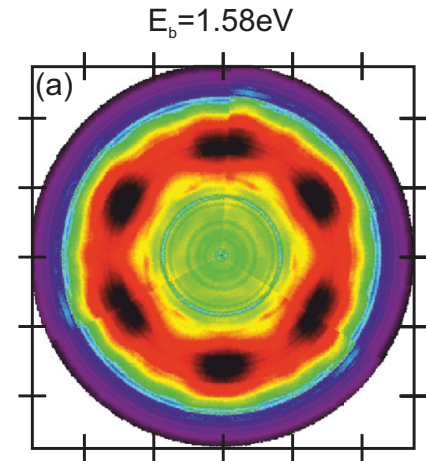

PTCDA HOMO

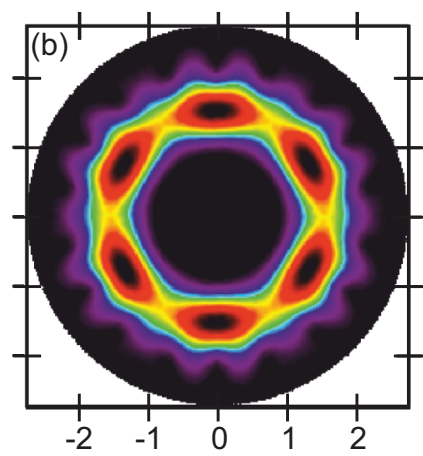

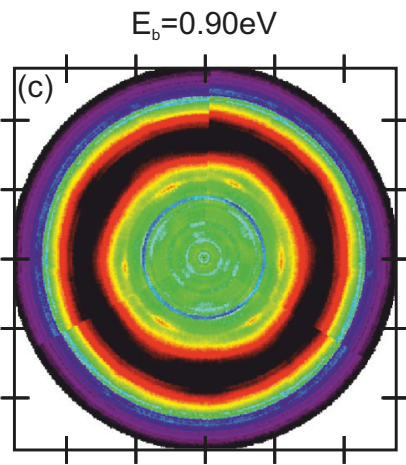

$\mathrm{CuPc} \mathrm{HOMO}$

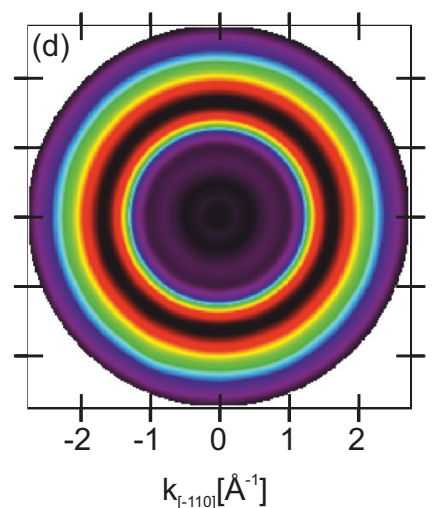

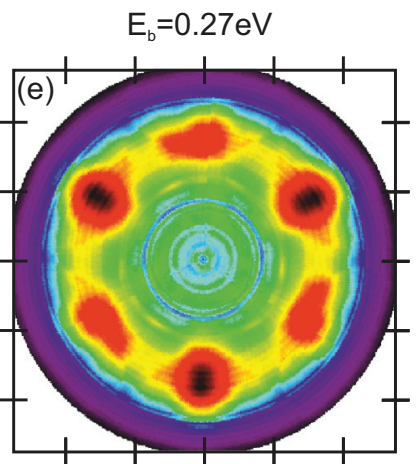

PTCDA LUMO

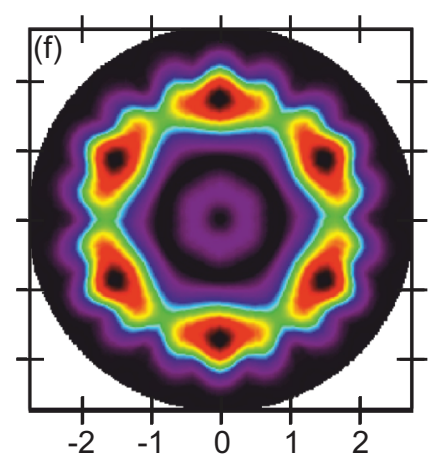

FIG. 1. (Color) Top row (a), (c), and (e): Constant binding energy maps obtained from ARPES for the three relevant binding energies of $\approx 0.7 \mathrm{ML} \mathrm{CuPc}$ on PTCDA/Ag(111). Bottom row (b), (d), and (f): Corresponding calculated CBEs for the PTCDA HOMO and LUMO as well as the CuPc HOMO. For details see text.
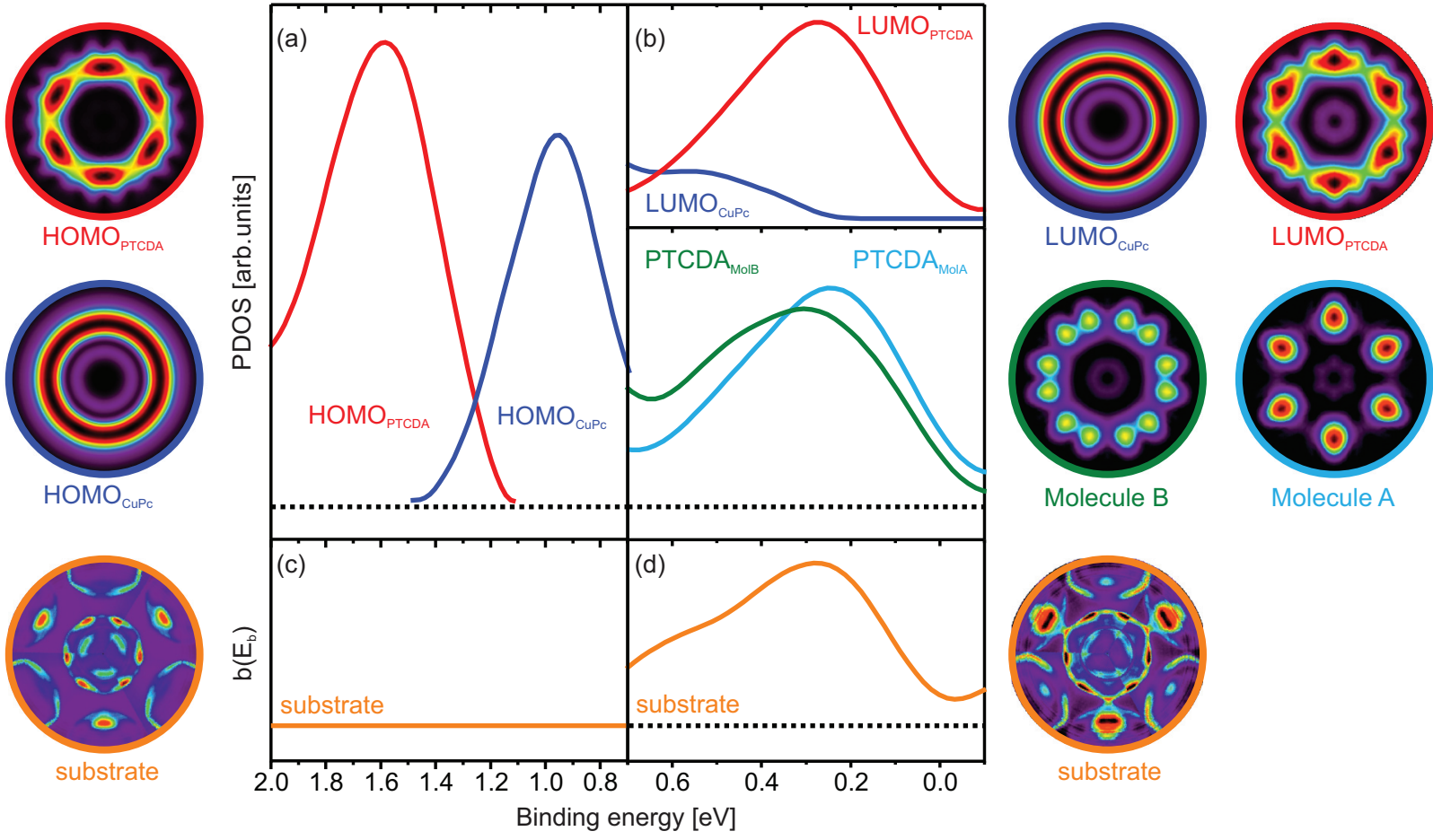

Molecule B

Molecule A

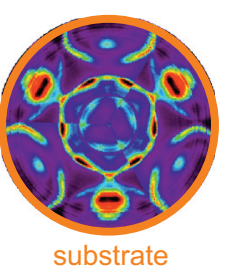

FIG. 2. (Color) (a), (b) Projected density of states (PDOS, $\left.a_{i}\left(E_{\mathrm{b}}\right)\right)$ and (c), (d) substrate contributions $b\left(E_{\mathrm{b}}\right)$ obtained from the orbital tomography analysis of the ARPES data. The momentum maps used in the fitting procedure are shown on the left and right side of the PDOS and the substrate amplitude. These momentum maps were calculated for the molecular orbitals and experimentally obtained for the substrate contribution. The CuPc coverage is $\approx 0.7 \mathrm{ML}$. 


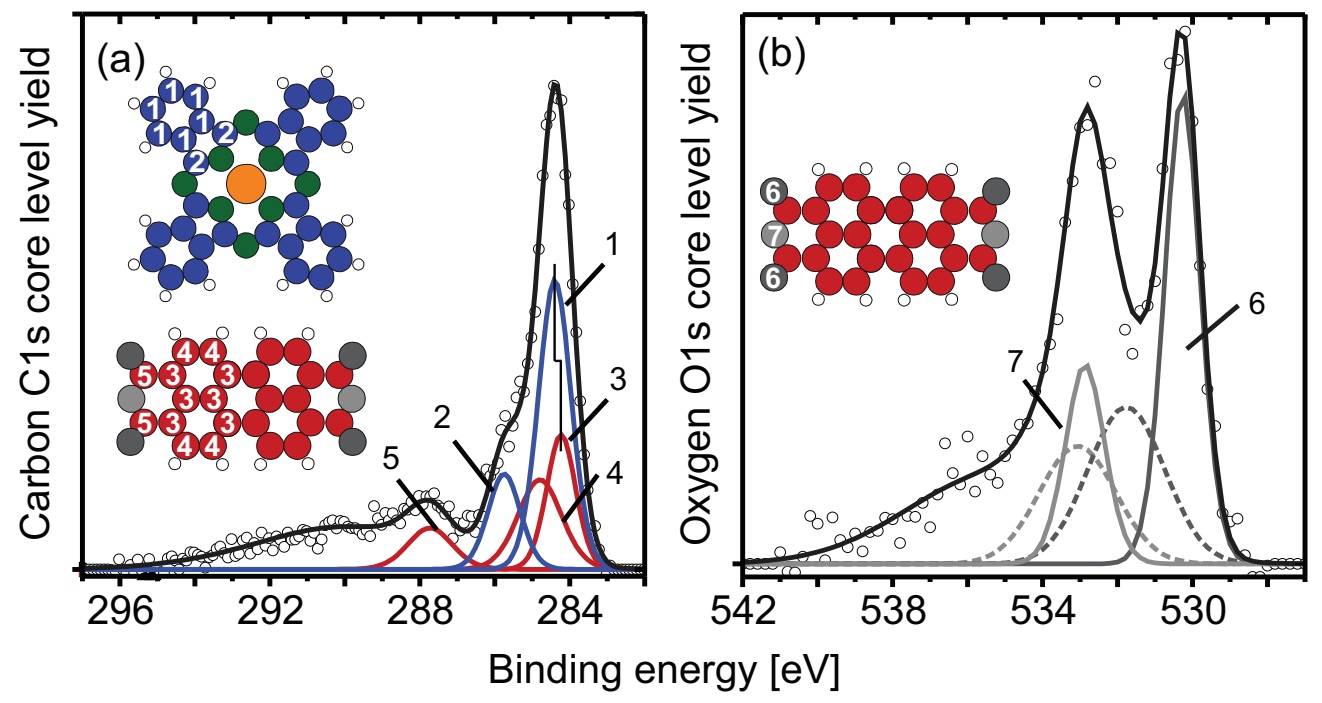

FIG. 3. (Color) Background subtracted core-level spectra for $\mathrm{C} 1 s$ and $\mathrm{O} 1 s$ emission lines recorded for 0.60 ML CuPc on PTCDA/ Ag(111). Measured data points are shown as circles, the corresponding best fit as a black line. The relevant core-level components used in the fitting model are shown as solid lines with a color code corresponding to the molecules shown as insets.

the substrate contribution factor $b\left(E_{B}\right)$ in Figs. 2(c) and 2(d). The emission patterns used for fitting are shown on the left and right side of the corresponding PDOS and the substrate amplitude b $\left(E_{\mathrm{b}}\right)$. The left column Figs. 2(a) and 2(c) shows the HOMO energy region modeled by the two emission patterns of the CuPc and PTCDA HOMOs [see also Figs. 1(d) and 1(f)]. Note that only the relevant $k$-space region was used for the fitting, and that in this case no emission features of the silver substrate are visible in this range. The contribution of the substrate could therefore be constrained to zero for the fitting. The resulting PDOS [Fig. 2(a)] reveals two well-separated peaks with their maxima at $E_{B}=1.58 \mathrm{eV}$ and $E_{B}=0.90 \mathrm{eV}$ for the PTCDA and CuPc HOMO, respectively. These values agree almost perfectly with the peak positions obtained from our earlier UPS study [30].

However, more importantly, the orbital tomography analysis also answers our most important question, namely the one for the nature of the UPS peak at the Fermi edge and the origin of its energetic shift upon $\mathrm{CuPc}$ adsorption. In a first step we modeled the CBE in question [Fig. 1(e)] with a superposition of the emission patterns of the PTCDA and $\mathrm{CuPc}$ LUMOs. In contrast to the HOMO region, the contribution of the substrate signal cannot be neglected here, as can be seen from the threefold symmetry of the experimental CBE map. The result of this first step is shown in the top panel of Fig. 2(b): The red and blue curves represent the PDOS of the PTCDA and CuPC LUMOs, respectively. In Fig. 2(d) the corresponding substrate contribution is shown. It is obvious that the PDOS of the PTCDA LUMO (red curve) is dominant while that of $\mathrm{CuPc}$ (blue curve) is essentially zero, in particular in the region close to $E_{F}$. The small increase in the intensity of the blue curve at binding energies above $0.4 \mathrm{eV}$ does not stem from the $\mathrm{CuPc}$ LUMO, but can be attributed to the low-binding energy tail of the CuPc HOMO peak. This artifact of the fitting procedure occurs since the HOMO and LUMO PDOS of CuPc for the disordered CuPc layer are very similar, see insets of Figs. 2(a) and 2(b). We can therefore conclude that the state at the Fermi edge is merely caused by the PTCDA LUMO and does not contain any contribution of the CuPc LUMO. Consequently, the electronic valence structure reveals no signs for any charge transfer into the CuPc LUMO or any hybridization of $\mathrm{CuPc}$ and PTCDA states. Nevertheless, the significant shift of the PTCDA LUMO found in standard UPS experiments [30] is confirmed by our orbital tomography analysis of the ARPES data, and with it the enhancement of the bonding between PTCDA and the silver surface that is caused by the formation of a heteromolecular bilayer film.

The absence of any $\mathrm{CuPc}$ contribution to the near- $E_{F}$ region allows a second step in the orbital tomography analysis: A deconvolution of the contributions from the two inequivalent PTCDA molecules in the unit cell (entitled A and B in the following). In the bottom part of Fig. 2(b) the PDOS resulting from an analysis with only the two separated emission pattern for the PTCDA molecules A and B, and a substrate contribution is displayed. The corresponding theoretical CBE maps are shown in the inset and discussed in more detail in Ref. [32]. The maximum in the PDOS for molecule B is found at larger binding energies than that of molecule A, which is similar to the situation in the pristine PTCDA monolayer film [32,38]. However, the energy difference between both LUMO levels is significantly reduced, from $170 \mathrm{meV}$ for the homomolecular structure to $110 \mathrm{meV}$ in the present case. This is due to the fact that the LUMO with the lower binding energy (that is that of molecule $\mathrm{A}, E_{B}=0.13 \mathrm{eV}$ in the homomolecular phase) shows a larger shift, namely $100 \mathrm{meV}$, in contrast to only $40 \mathrm{meV}$ found for the LUMO of molecule B $\left(E_{B}=0.30 \mathrm{eV}\right.$ in the homomolecular phase). In other words: Regarding the filling of their LUMOs, the molecule with the less strongly filled LUMO catches up with the other one so that the asymmetry in the LUMO filling between both molecules is reduced, although not lifted.

Finally we would like to mention that for both tomographic analyses of the LUMO energy region very similar behaviors of the substrate contribution $b\left(E_{B}\right)$ were observed [Fig. 2(d)]. In both cases a line shape rather similar to the PDOS of the PTCDA LUMOs was found. This behavior was not observed 
for a pristine PTCDA monolayer film. One might interpret this as an indication for a resonant transmission of substrate electrons caused by the formation of the CuPc/PTCDA bilayer film. But since it is found only for the LUMO, not for the HOMO region, it might also be attributed to a stronger hybridization of the PTCDA LUMO with substrate states exhibiting the intrinsic threefold symmetry of the substrate.

\section{B. Adsorption heights}

In this section we discuss in detail the results of NIXSW experiments for the $\mathrm{CuPc} / \mathrm{PTCDA} / \mathrm{Ag}(111)$ system performed for a $\mathrm{CuPc}$ coverage of $0.60 \mathrm{ML}$ at room temperature. We determined the adsorption yield profiles from the photoemission core levels (partial yield) $\mathrm{C} 1 s, \mathrm{O} 1 s, \mathrm{~N} 1 s$, and $\mathrm{Cu} 2 p$. By using a sophisticated fitting model for the $\mathrm{C} 1 s$ yield that is described in detail in the following subsection, we disentangle the signal from PTCDA and CuPc carbon species. Therefore we are able to determine the vertical adsorption geometry of the molecules (regarding both the average adsorption height and an eventual bending of the molecules) for PTCDA and CuPc separately, and hence - in particular-detect changes in the bonding of the PTCDA molecules caused by the CuPc adsorption. The latter is important, since the ARPES data discussed above suggest a modification of the PTCDA-Ag(111) bond depending on the CuPc coverage.

\section{Core-level spectra}

As already mentioned, we recorded XPS spectra from all species which are present in the organic bilayer film. While the $\mathrm{O} 1 s, \mathrm{~N} 1 s$, and $\mathrm{Cu} 2 p$ spectra contain contributions from one or two chemically different species stemming from only one molecular species, PTCDA or CuPc, the $\mathrm{C} 1 s$ spectra comprise photoelectrons from six different carbon species, two in the CuPc, and four in the PTCDA molecule. The XPS data fitting models are described in the following.

All spectra are background-subtracted by using a Shirley function [39]. Note that this type of background, which is more commonly used for treating bulk core-level spectra, has to be used in our case because of the photoelectron detection angle of almost $90^{\circ}$ with respect to the surface normal. In this emission direction the surface sensitivity of the PES experiment is strongly enhanced, but the attenuation of the photoelectrons is also much higher due to their longer pathway through the molecular film. A very welcome side effect of the increased surface sensitivity is the damping of the $\mathrm{Ag} 3 d$ plasmon peak which occurs in the N1s XPS scans, for the treatment of which a delicate background correction procedure had to be performed in earlier measurements performed in $45^{\circ}$ geometry $[11,40]$.

The $\mathrm{N} 1 s$ and $\mathrm{Cu} 2 p$ core-level spectra (not shown) consist of only one main peak accompanied by a small satellite structure at larger binding energies which has also been observed for the $\mathrm{CuPc}$ monolayer films on $\mathrm{Au}(111)$ and $\mathrm{Cu}(111)[21]$. A possible difference in the binding energies of the two nitrogen species could not be resolved.

The $\mathrm{O} 1 s$ and $\mathrm{C} 1 s$ spectra are more complex, exemplary data and the corresponding fitting models are shown in Fig. 3. Red and blue curves indicate the contributions of PTCDA and $\mathrm{CuPc}$ carbon species, respectively. The $\mathrm{CuPc}$ contributions consist
TABLE I. Parameters for the fitting of $\mathrm{C} 1 s$ and $\mathrm{O} 1 s$ core-level spectra: Binding energies $E_{\mathrm{B}}$, peak widths FHWM, and relative intensities $I$ for all peaks and satellites of chemically different species.

\begin{tabular}{lccr}
\hline \hline species & $E_{\mathrm{B}}[\mathrm{eV}]$ & FWHM $[\mathrm{eV}]$ & $I[\%]$ \\
\hline CuPc C $_{\mathrm{C}-\mathrm{C}}(1)$ & 284.4 & 1.0 & 26 \\
CuPc C $_{\mathrm{C}-\mathrm{N}}(2)$ & $284.4+1.4$ & 1.0 & 8 \\
PTCDA C $_{\mathrm{C}-\mathrm{C}}(3)$ & $284.4-0.17$ & 1.1 & 16 \\
PTCDA C $_{\mathrm{C}-\mathrm{H}}(4)$ & $284.4+0.40$ & 1.5 & 16 \\
PTCDA C $_{\mathrm{C}-\mathrm{O}}(5)$ & $284.4+3.3$ & 1.7 & 8 \\
Energy loss tail & 290.0 & 6.0 & 26 \\
Carboxylic O main peak & 530.3 & 1.3 & 28 \\
Carboxylic O satellite & $530.3+1.5$ & 2.5 & 24 \\
Anhydride O main peak & $530.3+2.6$ & 1.3 & 14 \\
Anhydride O satellite & $530.3+3.0$ & 2.5 & 12 \\
Energy loss tail & 535.8 & 6.5 & 18 \\
\hline \hline
\end{tabular}

of two peaks labeled (1) and (2), arising from two chemically different carbon species, which are indicated in the inset. We constrain the energy difference between these peaks in our fitting model to the value determined for $\mathrm{CuPc}$ in the gas phase [37]. For PTCDA the situation is more complex. Detailed studies using high-resolution core-level spectroscopy reported four different carbon species [34,41], three of which have very similar binding energies and hence form an asymmetric main peak in the spectrum. The fourth carbon species, that with bonds to the oxygen atoms $\left(\mathrm{C}_{\mathrm{C}-\mathrm{O}}\right.$ atoms), exhibits a clearly higher binding energy $(\approx 4 \mathrm{eV})$. For reasons of simplicity and in order to limit the number of free fitting parameters we use only two peaks (3) and (4) for fitting the main emission line, and a third peak for fitting the $\mathrm{C}_{\mathrm{C}-\mathrm{O}}$ peak (5). The energy difference between these peaks is also constrained during the fitting, based on earlier high-resolution XPS experiments [27].

Our fitting model correctly reproduces the line shape of the reference spectrum and is consequently used for the analysis of the NIXSW data. During NIXSW fitting we additionally constrain the relative intensities of all peaks stemming from the same molecular species [i.e., (1):(2) for $\mathrm{CuPc}$ and (3):(4):(5) for PTCDA]. In essence, this means that we neglect any bending of the carbon core of both molecules, for the benefit of a smaller number of fit parameters and a more stable fitting. Note that we have also tested other core-level fitting models with slightly different peak positions and different constraints for the intensity ratio of main and satellite peaks, but none of them resulted in higher coherent fractions $F^{H}$ for the carbon species in CuPc and PTCDA. This indicates that the assignment of the individual peaks to the CuPc and PTCDA molecules is correct (see discussion below). Very similar models have been successfully used earlier for studying other blends consisting of Phthalocyanine and PTCDA or similar molecules [23,29]. All optimized parameters of the fitting models are summarized in Table I. ${ }^{1}$

\footnotetext{
${ }^{1}$ Note that the intensities of the PTCDA main lines (3) and (4) do not reflect the stoichiometry of the corresponding carbon atoms in the molecule. However, since we did not resolve different carbon species of the same molecule in the NIXSW analysis, this small inaccuracy does not affect the results.
} 
TABLE II. NIXSW fit results for $0.6 \mathrm{ML} \mathrm{CuPc}$ on PTCDA/Ag(111). The numbers are averaged values from the analysis of all individual NIXSW scans. For $\mathrm{C}_{1} \mathrm{~s}_{\text {sum }}$ and $\mathrm{O} 1 \mathrm{~s}_{\text {sum }}$ the total photoelectron yields of the corresponding atomic species were analyzed.

\begin{tabular}{llcc}
\hline \hline & \multicolumn{1}{c}{$P^{H}$} & $D^{H}[\AA]$ & $F^{H}$ \\
\hline $\mathrm{C} 1 s_{\text {sum }}$ & $0.337(11)$ & - & $0.23(1)$ \\
$\mathrm{C} 1 s_{\text {CuPc }}$ & $0.550(10)$ & $6.02(2)$ & $0.67(3)$ \\
$\mathrm{N} 1 s_{\text {CuPc }}$ & $0.581(8)$ & $6.09(2)$ & $0.75(3)$ \\
$\mathrm{C} 42 p_{\text {CuPc }}$ & $0.566(15)$ & $6.06(4)$ & $0.89(4)$ \\
$\mathrm{C} 1 s_{\text {PTCDA }}$ & $0.170(10)$ & $2.76(2)$ & $0.63(3)$ \\
$\mathrm{O} 1 s_{\text {sum }}$ & $0.173(13)$ & $2.76(2)$ & $0.62(1)$ \\
$\mathrm{O} 1 s_{\text {carbox }}$ & $0.142(8)$ & $2.68(2)$ & $0.61(5)$ \\
$\mathrm{O} 1 s_{\text {anhy }}$ & $0.210(10)$ & $2.84(2)$ & $0.71(6)$ \\
\hline \hline
\end{tabular}

The O1s core-level spectrum [Fig. 3(b)] consist of two rather well separated peaks and a shoulder at the high binding energy side. The two peaks are attributed to two chemically different oxygen species in the PTCDA molecule, the carboxylic and the anhydride oxygen atoms (with smaller and larger binding energy, respectively). The fact that the two peaks do not reflect the stoichiometric ratio $2: 1$ and the shoulder on the high binding energy side of the spectra indicate that both peaks have (rather complex) satellite structures [4244]. The corresponding fitting model is shown in Fig. 3(b) with solid and dashed lines representing the main peaks (1) and (2), and the satellites, respectively. Regarding a separate NIXSW analysis for both oxygen species, in particular, the similar peak positions of the carboxylic satellite and the anhydride main peak have to be taken care of, since all contributions from both species have to be separated. We therefore constrain the energy difference between the main peaks (1) and (2) (the value was adapted from the O1s fitting model for the pristine PTCDA monolayer film on $\mathrm{Ag}(111)$ [43]) as well as the relative intensities of the peaks, which were fixed to the stoichiometric ratio of $2: 1$. The results shown in Fig. 3(b) represent the best fit for the O1s spectra. The model is quite similar to that obtained for the PTCDA low temperature structure [43], but differs from the RT model [43]. Since PTCDA/Ag(111) has a shorter bonding height (i.e., a stronger interaction) at LT rather than at RT, this finding can be taken as a first hint on an enhanced interaction of PTCDA with the silver surface also due to $\mathrm{CuPc}$ adsorption.

The NIXSW partial yield curves for carbon and oxygen are obtained from the fitting models introduced above. All parameters are listed in Table I, including all constraints for relative intensities of peaks contributing to the same partial yield. For carbon, these are all peaks from species of the same molecule, as mentioned above. For oxygen, the satellite peaks are constrained to their main peaks, individually for both species. Furthermore, all binding energy positions and peak widths are constrained to the values listed in the table.

\section{Vertical adsorption geometry}

In Figs. 4 and 5 we present the NIXSW results for the heteromolecular bilayer films. Typical photoelectron yield curves of individual NIXSW scans are shown in Fig. 4 for all chemical species that we differentiated. Error bars are based on a Monte Carlo error analysis implemented in CASAXPS [44,45],
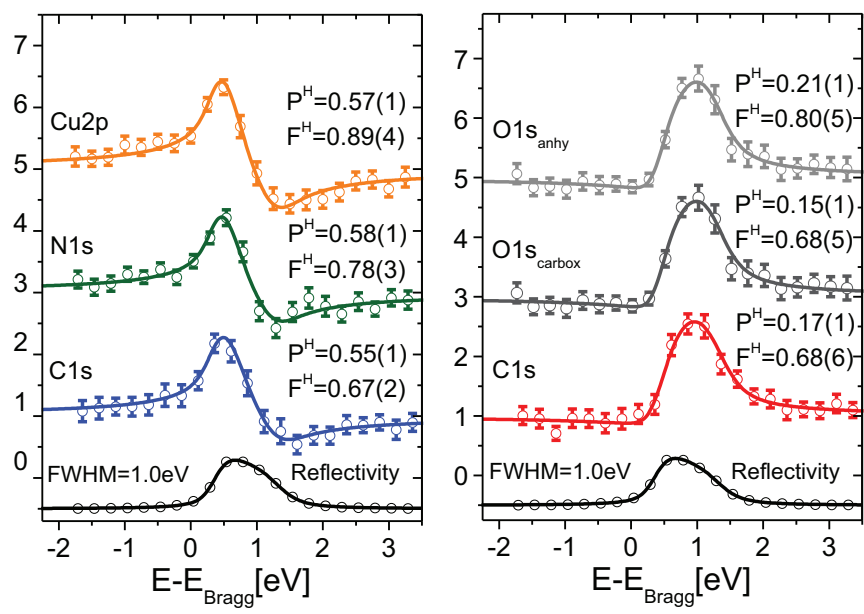

FIG. 4. (Color) Partial yield curves of single XSW scans for all distinguished species in the heteromolecular CuPc/PTCDA bilayer film, and reflectivity profiles of the substrate crystal (lowermost curves). Solid lines represent the best fit to the data obtained with TORRICELLI [45], the corresponding results (coherent position $P^{H}$ and fraction $F^{H}$ ) are given for each individual XSW scan. The error bars of the yield curves are calculated by a Monte Carlo error analysis included in CasaXPS [44,45]. The color code corresponds to the ball-and-stick models shown as insets in Fig. 3.

the software we use for fitting the core-level spectra. The yield curves are fitted using the XSW analysis software TORRICELLI [45] and the resulting fit parameters, coherent position $P^{H}$ and coherent fraction $F^{H}$, are also shown in the figure for these individual scans. Note that we have recorded several XSW scans for the same atomic species on different spots on the sample, which allows us to estimate the error in the coherent parameters. The results of all individual scans are plotted in an Argand diagram as data points (Fig. 5, small

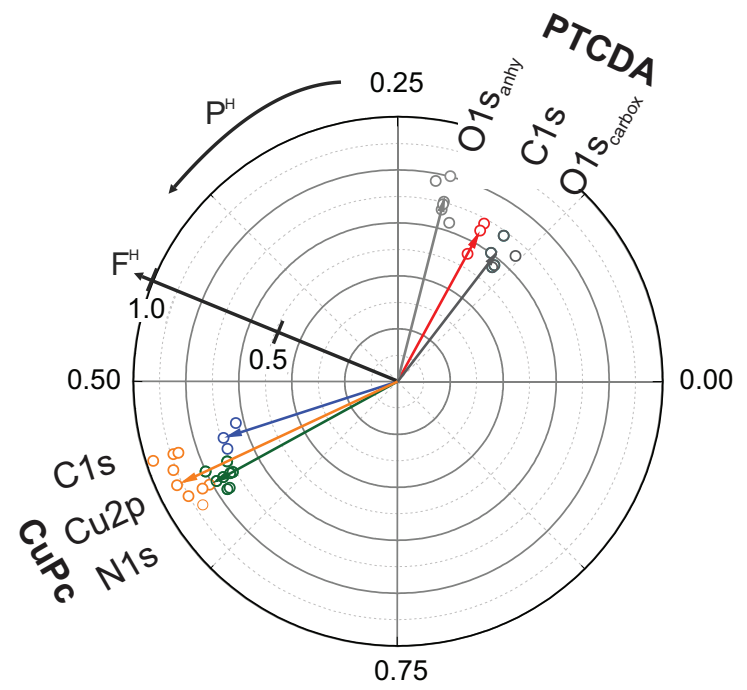

FIG. 5. (Color) Argand diagram illustrating the NIXSW fitting results for each individual NIXSW scan (colored circles) and the mean values of all scans for each species (colored vectors). Coherent positions $P^{H}$ and fractions $F^{H}$ are represented by the polar angle and the vector length, respectively. The color code is identical to that used for the yield curves (Fig. 4) and the ball-and-stick models (insets of Fig. 3). 
colored circles). Arrows of the same color mark the average of the individual results for one species, the scattering of the data points around their mean values is a quantitative measure for their statistical error (standard deviation of the results of the individual scans). This resulted in $\left|\Delta D^{H}\right|<0.02 \AA$ for the adsorption height of all species except for $\mathrm{Cu}$, which had a rather poor signal-to-noise ratio. Note that from the Argand diagram it is immediately clear that CuPc and PTCDA are located in different adsorption layers, since all results for atomic species of one molecular type are located close to each other, but well separated from those of the other molecule. All fit parameters are also listed in Table II.

Besides an estimation of statistical uncertainties one should also consider possible reasons for systematic errors in the data analysis, e.g., by incorrect core-level fitting models. For the copper, nitrogen and oxygen species the NIXSW data evaluation is straightforward, since these species show isolated or well-separated core-level peaks for chemically different species. Hence, the risk of undetected systematic errors is small. The analysis of the carbon data, however, is more complex and incorrect fitting models for the core-level data would immediately introduce (possibly) large systematic errors. As mentioned above, we have for this reason carefully checked our fitting model and tested it by comparing with alternative models. The indications for the correctness and reliability of our model, and consequently of the carbon-based NIXSW results, are: (i) The carbon adsorption heights are very close to that of the other species of the same molecule, as expected from the molecular structure. (ii) The present XPS fitting model yielded higher coherent fractions than other models. Any model with incorrect assignments of peaks (i.e., when a CuPc signal is partly attributed to a PTCDA carbon species, or vice versa) would immediately cause much lower coherent fractions, in particular in our case since the different molecular species are located in different layers and hence at very different adsorption heights. (iii) The coherent fractions for carbon are not much smaller than those of the other species. The difference is not larger than for comparable homomolecular systems and can easily be attributed to the higher number of atoms causing a slight variation in the adsorption height of the individual carbon atoms. In particular, it could be caused by a slight bending of the carbon backbone of the molecules that is neglected in out fitting model in order to limit the number of free parameters.

From the adsorption heights obtained by the NIXSW analysis we set up the adsorption model shown in Fig. 6. The PTCDA molecule is drawn in side view along its long molecular axis, CuPc is just shown in a schematic representation of its individual atoms. For the heteromolecular film the atoms are represented as colored circles, and gray circles indicate the vertical adsorption geometry of the PTCDA molecule in the pristine monolayer structure [43], i.e., without CuPc adsorbed on top. ${ }^{2}$

\footnotetext{
${ }^{2}$ Note that, since the adsorption height $D^{H}$ can only be determined modulo the bulk lattice spacing from the measured coherent fraction $P^{H}$, the experimental result in principle could also represent an adsorption height of approx. $3.7 \AA$ instead of $6.05 \AA$. However, $3.7 \AA$
}

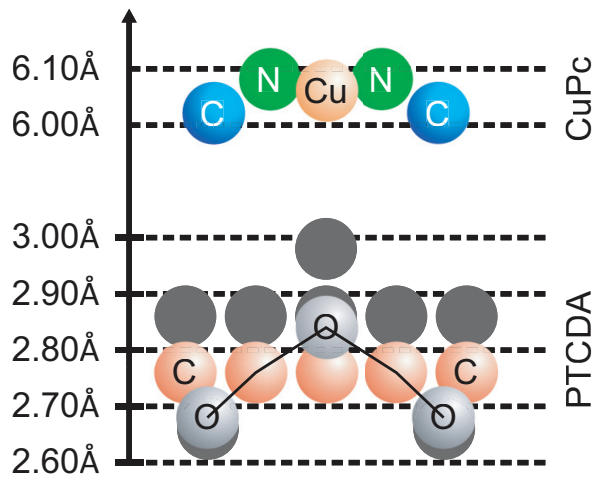

FIG. 6. (Color) Model for the vertical adsorption geometry of the CuPc/PTCDA bilayer film for $\Theta_{\mathrm{CuPc}}=0.60 \mathrm{ML}$ and $\Theta_{\mathrm{CuPc}}=$ $0.95 \mathrm{ML}$ at room temperature. The uppermost Ag layer is located at $0.0 \AA$. Colored circles mark the adsorption heights of the atomic species of both molecules in the bilayer film, gray circles indicate the vertical atomic positions in the pristine PTCDA monolayer film [43], i.e., for $\Theta_{\mathrm{CuPc}}=0 \mathrm{ML}$.

Similar to the adsorption on various noble metal surfaces [11,21], the CuPc molecules exhibit an almost perfectly flat adsorption geometry on PTCDA with an intermolecular distortion smaller than $0.1 \AA$. In contrast, PTCDA is significantly distorted, similar to the case of the PTCDA monolayer structure on $\operatorname{Ag}(111)$ : The carboxylic oxygen atoms approach the silver surface while the anhydride oxygen atoms are shifted to a position above the molecular backbone. This finding, and the overall adsorption height of PTCDA, indicates a chemical interaction between the molecule and the silver surface as it is known from the homomolecular adsorbate structure $[11,43,44,46]$. However, in the bilayer structure the vertical distance between both oxygen species is reduced by a factor of 2 compared to the PTCDA monolayer film, i.e., the bending of the molecule is reduced by the on-top adsorption of CuPc. This is mainly due to a down shift of the anhydride oxygen atoms, while the carboxylic atoms hardly move. Also the carbon backbone is found at a position $0.1 \AA$ lower, an effect that was also seen in $a b$ initio calculations [47]. These findings clearly indicate a shorter bond of the $\pi$-conjugated part of the molecule with the silver surface in the bilayer, which is in perfect agreement with the additional charge transfer from the substrate into the PTCDA LUMO observed by ARPES. As discussed by Willenbockel et al. [6] this behavior represents the normal coupling between electronic and geometric structure for weakly bonded molecular adsorbate systems: An upward shift of the LUMO binding energy and a shorter adsorption height are considered as electronic and geometric fingerprint, respectively, of a stronger bonding between substrate and adsorbate. Furthermore, the bonding of the carboxylic oxygen to the Ag substrate appears to be essentially unchanged, which is in agreement with the fact that the lateral structure-and herewith the adsorption site of the PTCDA molecules, is unchanged. We can conclude that

are neither realistic for a first-layer, nor for a second-layer molecule. It is therefore obvious that $\mathrm{CuPc}$ is adsorbed in the second layer with a distance of $\approx 6.05 \AA$ to the silver surface. 
TABLE III. Interlayer distances of the $\mathrm{CuPc}$ atomic species to the PTCDA carbon backbone $\triangle D_{\mathrm{C}}^{H}$ and to the PTCDA anhydride oxygen atoms $\Delta D_{\mathrm{O}-\text { anhy }}^{H}$, respectively. The distance are normalized to the sum of the corresponding van der Waals radii $r_{C}=1.77 \AA$, $r_{N}=1.55 \AA, r_{C u}=1.40 \AA$, and $r_{O}=1.50 \AA$. [48].

\begin{tabular}{lcc}
\hline \hline & $\Delta D_{\mathrm{C}}^{H}[\%]$ & $\Delta D_{\mathrm{O}-\text { anhy }}^{H}[\%]$ \\
\hline Carbon $_{\mathrm{CuPc}}$ & $92(2)$ & $97(2)$ \\
Nitrogen $_{\mathrm{CuPc}}$ & $100(2)$ & $107(2)$ \\
Copper $_{\mathrm{CuPc}}$ & $104(2)$ & $111(2)$ \\
\hline \hline
\end{tabular}

both geometric and electronic structure indicate a stronger interaction of PTCDA with the silver surface caused by the formation of the bilayer film with $\mathrm{CuPc}$.

We would like to mention that the coherent fraction of the PTCDA carbon species also changes upon $\mathrm{CuPc}$ adsorption. In the pristine PTCDA/Ag(111) layer we found coherent fractions for the carbon species that were approximately $10 \%$ larger than those for the bilayer structure. Although this change is still within the experimental uncertainty, it might be an indication for a small vertical disorder in the PTCDA monolayer film induced by $\mathrm{CuPc}$ adsorption.

The CuPc molecules adsorb at a height of $\approx 6.05 \AA$, about $\approx 3.3 \AA$ above the PTCDA backbone. In order to evaluate this spacing between the two layers in terms of interaction strength we list these distances in units of the corresponding sum van der Waals radii of the atomic species involved. Van der Waals radii are taken from Ref. [48]. These normalized distances $\Delta D_{\mathrm{C}}^{H}$ are listed in Table III. While the distance of the nitrogen and copper atoms to the PTCDA backbone is equal or larger than the sum of the van der Waals radii, a small overlap of the radii is found for the carbon atoms of both molecules (distance of $92 \%$ ). This is not only due to a larger van der Waals radius of carbon compared to copper and nitrogen, but also caused by a small bending of the CuPc carbon atoms towards the PTCDA backbone. We also calculated the normalized distances $\Delta D_{\mathrm{O}-\text { anhy }}^{H}$ of the atoms of CuPc to the anhydride oxygen of PTCDA, see last column of Table III. All these values are larger than the corresponding $\Delta D_{\mathrm{C}}^{H}$ values, although the anhydride oxygens are located above the carbon backbone of PTCDA.

This analysis indicates that the distance between the PTCDA and the CuPc layer is defined by the contact of the carbon backbones of both molecules (in the sense of an equilibrium of repulsive and attractive forces), not by the other species. The bonding of copper, nitrogen, and oxygen to the carbon atoms of the other molecule, or even $\mathrm{Cu}-\mathrm{O}$ or $\mathrm{N}-\mathrm{O}$ bonds do not play a significant role, since the distances between these species (in units of the van der Waals radii) are larger. We can further conclude that the interaction between $\mathrm{CuPc}$ and PTCDA is mainly dominated by van der Waals forces for this low $\mathrm{CuPc}$ coverage. This is based on the normalized value of $92 \%$ of the C-C van der Waals distance for the PTCDA-CuPc layer spacing, which agrees very well with interlayer spacings

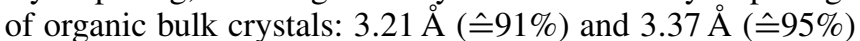
were reported for PTCDA [49] and the $\operatorname{CuPc} \beta$ phase [50], respectively. This indicates that the interlayer interaction of our mixed bilayer system and in organic bulk crystals is of very similar strength, and hence mainly caused by van der Waals forces.

\section{DISCUSSION}

Our comprehensive investigation of the geometric and electronic structure of the heteromolecular bilayer system $\mathrm{CuPc}$ on PTCDA/Ag(111) allows new insights in the fundamental interaction mechanisms in these films. At room temperature CuPc behaves like a dilute 2D gas on PTCDA, the density of which rises continuously with increasing $\mathrm{CuPc}$ coverage. This in turn leads to a continuously increasing, additional population of the PTCDA LUMO, while the CuPc LUMO remains unfilled, as determined by UPS [30] and ARPES (see above). At the same time the PTCDA-Ag adsorbate distance is reduced. Hence, just due to the rising density of $\mathrm{CuPc}$ molecules in the second layer, the charge transfer from the silver surface to the first (PTCDA) layer increases, while the bonding distance between the two decreases, both indicating a stronger Ag-PTCDA interaction. The additional charge transfer into the PTCDA LUMO can be explained by an additional screening mechanism, which is introduced by the adsorption of CuPc on PTCDA. The second adsorbate layer represents a polarizable medium in close vicinity to the PTCDA molecules, which is able to screen the additional charge in the PTCDA molecule (namely in its LUMO). This energetically stabilizes the adsorbate system, which in this case manifests itself in an increased binding energy of the PTCDA LUMO. According to the conjunction of electronic and geometric structure of organic adsorbates (that is, stronger binding goes along with shorter binding distance $[6,22]$ ) this is correlated to the observed decrease in the PTCDA adsorption height. In the following two paragraphs we explain the formation of the additional screening mechanism introduced by the CuPc layer in more detail.

In case of adsorption of only one molecular layer on a metal surface, the question whether or not a charge transfer into the molecules is favorable will be determined by the energy gain (or loss) caused by all charge redistributions at the interface, including all screening effects. For a pristine PTCDA monolayer film on $\operatorname{Ag}(111)$ the result is an effective net charge transfer of 0.31 electrons into each PTCDA molecule [51]. Consequently the PTCDA LUMO becomes partially occupied (with an even higher amount of charge, since the net charge transfer also includes back donation from the molecule to the silver). The amount of effective charge on the molecule depends on an equilibrium between the energy gain caused by the charge transfer, and the energetic costs of intermolecular and intramolecular Coulomb repulsions. This becomes obvious when we consider a freestanding PTCDA monolayer of neutral molecules. Placing a certain amount of charge on each PTCDA molecule results in an intramolecular Coulomb repulsion between the added charge and the electronic system of the molecule. This effect increases the total energy of the freestanding layer by an energy $U$ per molecule. ${ }^{3}$ The intermolecular Coulomb repulsion between

\footnotetext{
${ }^{3}$ This is the analog to the on-site Hubbard- $U$, which is well known for the case of charging a singly occupied (molecular) orbital [53].
} 
charges on neighboring molecules leads to additional energetic costs $V$. Both energies $U$ and $V$ influence the charge transfer into each molecule and the binding energies of the molecular orbitals. On a metal surface, beside charge redistributions in the entire adsorbate system, the effective charge on each molecule can be screened by image charges, which are created in the metal substrate. This reduces the intramolecular and intermolecular energetic costs $U$ and $V$, and leads to a lower total energy for the adsorbate system. In the end the charge transfer into a molecule will be favored when the energy gain by all charge redistributions (including the screening) is larger than the energetic costs $U$ and $V$.

In the CuPc/PTCDA bilayer film, the effective charges in the PTCDA layer are not only screened by image charges in the metal, but also by the polarizability of the $\mathrm{CuPc}$ molecules in the second layer. For any chosen molecule with an intrinsic polarizability $\alpha$ the efficiency of the molecular screening depends on the density of molecules in the second layer $N$ and the interlayer spacing $R[52,53]$. This additional screening effect lowers both intramolecular and intermolecular Coulomb repulsion energies $U$ and $V$, and creates a new equilibrium between the energy gained from adsorbing the molecules on the surface (caused by the charge redistributions) and the energy losses $U$ and $V$. As a consequence, additional charge transfer into the PTCDA molecule may become energetically favorable. For the CuPc/PTCDA bilayer film, the continuous population of the PTCDA LUMO is the result of the $\mathrm{CuPc}$ growth behavior on PTCDA. In the 2D gas phase, the $\mathrm{CuPc}$ density-and hence the efficiency of the molecular screening-increases continuously with $\mathrm{CuPc}$ coverage. This in turn continuously modifies the energy balance between the adsorption energy gain and costs, and consequently allows more charge transfer into the PTCDA LUMO. This additional charge redistribution between silver surface and PTCDA indicates a stronger chemical interaction at the metal-organic interface, in perfect agreement with the smaller adsorption height of PTCDA (compared to its pristine monolayer structure) that we found in NIXSW [6].

\section{SUMMARY}

In this paper, we have studied the correlation between electronic and geometric structure (namely charge transfer and bonding distances) at the organic-organic and the metalorganic interfaces of the CuPc/PTCDA bilayer adsorbate system on $\operatorname{Ag}(111)$. Two main experimental results were obtained: (i) The weak chemisorptive interaction between
PTCDA and the $\operatorname{Ag}(111)$ surface is strengthened by the adsorption of $\mathrm{CuPc}$ in the second layer. This is surprising, since CuPc itself only physisorbs on the PTCDA/Ag(111) system. This finding is based on earlier UPS results [30] showing a continuous shift of the Fermi-level peak towards higher binding energies due to CuPc adsorption (which was confirmed in this work), and ARPES measurements, which prove that this peak exclusively represents the PTCDA LUMO and does not contain any contribution of a $\mathrm{CuPc}$ state. (ii) The PTCDA-Ag(111) distance, which is compatible with weak chemisorption, is significantly reduced by the adsorption of $\mathrm{CuPc}$, as seen in NIXSW. The CuPc-PTCDA distance, however, is comparable to typical interlayer spacings in molecular crystals and hence indicates physisorption. These findings are in excellent agreement with the UPS and ARPES results: They indicate that the interaction between the organic layers is mainly driven by van der Waals forces, and that the chemisorptive character of the PTCDA-Ag(111) bond increases with increasing $\mathrm{CuPc}$ coverage. The significant reduction of the PTCDA adsorption height and the additional charge transfer into the PTCDA LUMO are geometric and electronic fingerprints of that increasing bonding strength.

These findings raise the question for the fundamental mechanism that strengthens the PTCDA-Ag bond when the CuPc molecules physisorb on top of the PTCDA/Ag(111) system. We suggest a model involving intermolecular screening of charged PTCDA molecules: The adsorbed CuPc molecules, since they represent a polarizable medium, enable an additional screening mechanism assisting the screening by the surface image charges. The intramolecular and intermolecular Coulomb repulsion caused by charging the PTCDA molecules can be compensated (at least partially) by this screening, which allows a more effective charge transfer into the PTCDA molecule. This explains the shift of the PTCDA LUMO towards higher binding energy, and thus also the reduced bonding distance.

Note added in proof. Recently, we became aware of the work of Gruenewald et al. [54], which addresses the aspect of interaction strength at the CuPc/PTCDA interface with complementary techniques.

\section{ACKNOWLEDGMENTS}

We thank G. Koller, D. Lüftner, and P. Puschnig for their support during ARPES data acquisition and analysis. We would also like to acknowledge support by the ESRF and BESSY staff during the beam times.
[1] L. Kilian, E. Umbach, and M. Sokolowski, Surf. Sci. 573, 359 (2004).

[2] F. S. Tautz, Prog. Surf. Sci. 82, 479 (2007).

[3] C. H. Schwalb, S. Sachs, M. Marks, A. Schöll, F. Reinert, E. Umbach, and U. Höfer, Phys. Rev. Lett. 101, 146801 (2008).

[4] J. Ziroff, F. Forster, A. Schöll, P. Puschnig, and F. Reinert, Phys. Rev. Lett. 104, 233004 (2010).

[5] S. Duhm, A. Gerlach, I. Salzmann, B. Braeker, R. Johnson, F. Schreiber, and N. Koch, Org. Electron. 9, 111 (2008).
[6] M. Willenbockel, D. Lüftner, B. Stadtmüller, G. Koller, C. Kumpf, S. Soubatch, P. Puschnig, M. G. Ramsey, and F. S. Tautz, Phys. Chem. Chem. Phys. 17, 1530 (2015).

[7] K. Manandhar, T. Ellis, K. T. Park, T. Cai, Z. Song, and J. Hrbek, Surf. Sci. 601, 3623 (2007).

[8] H. Karacuban, M. Lange, J. Schaffert, O. Weingart, T. Wagner, and R. Möller, Surf. Sci. 603, L39 (2009).

[9] C. Stadler, S. Hansen, I. Kröger, C. Kumpf, and E. Umbach, Nature Phys. 5, 153 (2009). 
[10] M. Häming, C. Scheuermann, A. Schöll, F. Reinert, and E. Umbach, J. Electron Spectrosc. Relat. Phenom. 174, 59 (2009).

[11] I. Kröger, B. Stadtmüller, C. Stadler, J. Ziroff, M. Kochler, A. Stahl, F. Pollinger, T.-L. Lee, J. Zegenhagen, F. Reinert et al., New J. Phys. 12, 083038 (2010).

[12] B. Stadtmüller, I. Kröger, F. Reinert, and C. Kumpf, Phys. Rev. B 83, 085416 (2011).

[13] A. Gerlach, T. Hosokai, S. Duhm, S. Kera, O. T. Hofmann, E. Zojer, J. Zegenhagen, and F. Schreiber, Phys. Rev. Lett. 106, 156102 (2011).

[14] C. Krull, R. Robles, A. Mugarza, and P. Gambaradella, Nature Mater. 12, 337 (2013).

[15] I. Kröger, P. Bayersdorfer, B. Stadtmüller, C. Kleimann, G. Mercurio, F. Reinert, and C. Kumpf, Phys. Rev. B 86, 195412 (2012).

[16] I. Kröger, B. Stadtmüller, C. Wagner, C. Weiss, R. Temirov, F. S. Tautz, and C. Kumpf, J. Chem. Phys. 135, 234703 (2011).

[17] J. Fraxedas, S. Garcia-Gil, S. Monturet, N. Lorente, I. Fernandez-Torrente, K. J. Franke, J. I. Pascual, A. Vollmer, R.-P. Blum, N. Koch et al., J. Phys. Chem. C 115, 18640 (2011).

[18] C. Braatz, G. Ohl, and P. Jakob, J. Chem. Phys. 136, 134706 (2012).

[19] G. Heimel, S. Duhm, I. Salzmann, A. Gerlach, A. Strozecka, J. Niederhausen, C. Brüker, T. Hosokai, I. Fernandez-Torrente, G. Schulze et al., Nature Chem. 5, 187 (2013).

[20] S. Soubatch, I. Kröger, C. Kumpf, and F. S. Tautz, Phys. Rev. B 84, 195440 (2011).

[21] I. Kröger, B. Stadtmüller, C. Kleimann, P. Rajput, and C. Kumpf, Phys. Rev. B 83, 195414 (2011).

[22] B. Stadtmüller, D. Lüftner, M. Willenbockel, E. Reinisch, T. Sueyoshi, G. Koller, S. Soubatch, M. G. Ramsey, P. Puschnig, F. S. Tautz et al., Nature Commun. 5, 3685 (2014).

[23] B. Stadtmüller, S. Schröder, F. C. Bocquet, C. Henneke, C. Kleimann, S. Soubatch, M. Willenbockel, B. Detlefs, J. Zegenhagen, T.-L. Lee et al., Phys. Rev. B 89, 161407 (2014).

[24] F. Sellam, T. Schmitz-Hübsch, M. Toerker, S. Mannsfeld, H. Pröhl, T. Fritz, K. Leo, C. Simpson, and K. Müllen, Surf. Sci. 478, 113 (2001).

[25] W. Chen, H. Huang, S. Chen, X. Y. Gao, and A. Wee, J. Phys. Chem. C 112, 5036 (2008).

[26] H. Huang, W. Chen, S. Chen, D. Qi, X. Gao, and A. Wee, Appl. Phys. Lett. 94, 163304 (2009).

[27] M. Häming, M. Greif, C. Sauer, A. Schöll, and F. Reinert, Phys. Rev. B 82, 235432 (2010).

[28] B. Stadtmüller, M. Gruenewald, J. Peuker, R. Forker, T. Fritz, and C. Kumpf, J. Phys. Chem. C 118, 28592 (2014).

[29] C. Kleimann, B. Stadtmüller, S. Schröder, and C. Kumpf, J. Phys. Chem. C 118, 1652 (2014).

[30] B. Stadtmüller, T. Sueyoshi, G. Kichin, I. Kröger, S. Soubatch, R. Temirov, F. S. Tautz, and C. Kumpf, Phys. Rev. Lett. 108, 106103 (2012).
[31] P. Puschnig, E.-M. Reinisch, T. Ules, G. Koller, S. Soubatch, M. Ostler, L. Romaner, F. S. Tautz, C. Ambrosch-Draxl, and M. G. Ramsey, Phys. Rev. B 84, 235427 (2011).

[32] B. Stadtmüller, M. Willenbockel, E.-M. Reinisch, T. Ules, F. C. Bocquet, S. Soubatch, P. Puschnig, G. Koller, M. G. Ramsey et al., Europhys. Lett. 100, 26008 (2012).

[33] P. Puschnig, S. Berkebile, A. J. Fleming, G. Koller, K. Emtsev, T. Seyller, J. D. Riley, C. Ambrosch-Draxl, F. P. Netzer, and M. G. Ramsey, Science 326, 702 (2009).

[34] Y.Zou, L. Kilian, A. Schöll, T. Schmidt, R. Fink, and E. Umbach, Surf. Sci. 600, 1240 (2006).

[35] D. Woodruff, Rep. Prog. Phys. 68, 743 (2005).

[36] J. Zegenhagen, Surf. Sci. Rep. 18, 202 (1993).

[37] F. Evangelista, V. Carravetta, G. Stefani, B. Jansik, M. Alagia, S. Stranges, and A. Ruocco, J. Chem. Phys. 126, 124709 (2007).

[38] M. Rohlfing, R. Temirov, and F. S. Tautz, Phys. Rev. B 76, 115421 (2007).

[39] D. Shirley, Phys. Rev. B 5, 4709 (1972).

[40] C. Stadler, S. Hansen, F. Pollinger, C. Kumpf, E. Umbach, T.-L. Lee, and J. Zegenhagen, Phys. Rev. B 74, 035404 (2006).

[41] A. Schöll, Y.Zou, M. Jung, T. Schmidt, R. Fink, and E. Umbach, J. Chem. Phys. 121, 10260 (2004).

[42] P. Unwin, D. Onoufriou, and T. Jones, Surf. Sci. 547, 45 (2003).

[43] A. Hauschild, R. Temirov, S. Soubatch, O. Bauer, A. Schöll, B. C. C. Cowie, T.-L. Lee, F. S. Tautz, and M. Sokolowski, Phys. Rev. B 81, 125432 (2010).

[44] G. Mercurio, O. Bauer, M. Willenbockel, N. Fairley, W. Reckien, C. H. Schmitz, B. Fiedler, S. Soubatch, T. Bredow, M. Sokolowski et al., Phys. Rev. B 87, 045421 (2013).

[45] G. Mercurio, Study of Molecule-Metal Interfaces by Means of the Normal Incidence X-ray Standing Wave Technique, Schriften des Forschungszentrums Jülich, Reihe Schlüsseltechnologien, Vol. 49, Ph.D. thesis, RWTH Aachen University, 2012, http://www.fz-juelich.de/zb/juwel.

[46] O. Bauer, G. Mercurio, M. Willenbockel, W. Reckien, C. Heinrich Schmitz, B. Fiedler, S. Soubatch, T. Bredow, F. S. Tautz, and M. Sokolowski, Phys. Rev. B 86, 235431 (2012).

[47] D. Egger, V. Ruiz, W. Saidi, T. Bucko, A. Tkatchenko, and E. Zojer, J. Phys. Chem. C 117, 3055 (2013).

[48] A. Bondi, J. Phys. Chem. 68, 441 (1964).

[49] S. R. Forrest and Y. Zhang, Phys. Rev. B 49, 11297 (1994).

[50] N. B. McKeown, Phthalocyanine Materials (Cambridge University Press, Cambridge, 1998).

[51] L. Romaner, D. Nabok, P. Puschnig, E. Zojer, and C. AmbroschDraxl, New J. Phys. 11, 053010 (2009).

[52] K. Boni and V. Kresin, Electric-Dipole Polarizabilities Of Atoms, Molecules, And Clusters (World Scientific, Singapore, 1997).

[53] I. Fernandez Torrente, K. Franke, and J. Pascual, J. Phys.: Condens. Matter 20, 184001 (2008).

[54] M. Gruenewald, C. Sauer, J. Peuker, M. Meissner, F. Sojka, A. Schöll, F. Reinert, R. Forker, and T. Fritz, Phys. Rev. B 91, 155432 (2015). 